PROCEEDINGS OF THE

AMERICAN MATHEMATICAL SOCIETY

Volume 140, Number 10, October 2012, Pages 3549-3555

S 0002-9939(2012)11200-6

Article electronically published on February 24, 2012

\title{
ON THE EXISTENCE OF $J$-CLASS OPERATORS ON BANACH SPACES
}

\author{
AMIR BAHMAN NASSERI
}

(Communicated by Thomas Schlumprecht)

\begin{abstract}
In this paper we answer in the negative the question raised by G. Costakis and A. Manoussos whether there exists a J-class operator on every non-separable Banach space. In particular we show that there exists a non-separable Banach space constructed by S. Argyros, A. Arvanitakis and A. Tolias such that the $J$-set of every operator on this space has empty interior for each non-zero vector. On the other hand, on non-separable spaces which are reflexive there always exists a $J$-class operator.
\end{abstract}

\section{Preliminaries AND THE MAin Result}

Let $X$ be a real or complex Banach space. If $X$ is a real Banach space, then by $X_{\mathbb{C}}$ we denote the complexification of $X$. By $L(X)$ we mean the space of all bounded linear operators acting on $X$. If $T \in L(X)$, the symbol $\sigma(T)$ stands for the spectrum of $T$. Consider any subset $C$ of $X$. The symbol $C^{\circ}$ denotes the interior of $C$ in the norm topology of $X$. The symbol $\operatorname{orb}(T, x)$ denotes the orbit of $x$ under $T$, i.e. $\operatorname{orb}(T, x):=\left\{T^{n} x: n \in \mathbb{N} \cup\{0\}\right\}$. If $X$ is separable and $\operatorname{orb}(T, x)$ is dense, then $T$ is called hypercyclic, which is equivalent to $T$ being topologically transitive; i.e. for every pair of non-empty open sets $U, V \subset X$, there exists a non-negative integer $n$ such that $T^{n}(U) \cap V \neq \emptyset$. Following [7, by $J_{T}(x)$ we denote the $J$-set of $x$ under $T$, i.e.

$$
\begin{array}{r}
J_{T}(x):=\{y \in X: \text { there exists a strictly increasing sequence } \\
\text { of natural numbers }\left(k_{n}\right) \text { and a sequence } \\
\left.\left(x_{n}\right) \text { in } X, \text { such that } x_{n} \rightarrow x \text { and } T^{k_{n}} x_{n} \rightarrow y\right\} .
\end{array}
$$

If $J_{T}(x)=X$ for some $x \in X \backslash\{0\}$, then $T$ is called a $J$-class operator. By $A_{T}$ we denote the set of all $x \in X$ such that $J_{T}(x)=X$. On separable spaces every hypercyclic operator is $J$-class, but the converse is not true. It is known [5] that on $l^{\infty}$, there does not exist a topological transitive operator. On the other hand there exist $J$-class operators such as the weighted backward shift $\lambda B: l^{\infty} \rightarrow l^{\infty}$, $\lambda B\left(x_{1}, x_{2}, \ldots\right):=\left(\lambda x_{2}, \lambda x_{3}, \ldots\right)$ for $|\lambda|>1$. Therefore it is natural to ask whether every non-separable Banach space admits a $J$-class operator [7]. Our main result is the following:

Received by the editors September 28, 2010 and, in revised form, April 13, 2011 and April 15, 2011.

2000 Mathematics Subject Classification. Primary 47A16; Secondary 37B99, 54H20.

Key words and phrases. J-class operators, hypercyclicity.

(C)2012 American Mathematical Society Reverts to public domain 28 years from publication 
Theorem 1.1. There exists a non-separable complex Banach space $X$ on which the $J$-set of every operator has empty interior for every non-zero vector. Consequently there exists no $J$-class operator on $X$.

The statement in Theorem 1.1 gives us a stronger result than the question raised by G. Costakis and A. Manoussos, since in general $\left(J_{T}(x)\right)^{\circ} \neq \emptyset$ does not imply that $J_{T}(x)=X$, 4. As is clear from our proof, the conclusion of Theorem 1.1 is satisfied for every complex non-separable Banach space, for which every $T \in L(X)$ is of the form $T=\lambda I+S$ with $\lambda \in \mathbb{C}$ and $S$ a strictly singular operator with separable range. A real non-separable HI (Hereditarily Indecomposable) Banach space containing no reflexive subspace for which every $T \in L(X)$ takes the form $T=\lambda I+S$ with $\lambda \in \mathbb{R}$ and $S$ a weakly compact operator with separable range has been constructed by S. Argyros, A. Arvanitakis and A. Tolias in [3]. The complexification of this space is easily shown to satisfy our requirements, and thus the conclusion of Theorem 1.1. In contrast we show in Theorem 1.18 that every non-separable reflexive Banach space admits a $J$-class operator.

Definition 1.2. Let $X, Y$ be infinite dimensional Banach spaces. A linear and bounded operator $S: X \rightarrow Y$ is called strictly singular if for every infinite dimensional subspace $M \subset X$ the restriction $S_{\mid M}: M \rightarrow S(M)$ is not an isomorphism (linear homeomorphism).

Remark 1.3. If $X=Y$, then an immediate consequence of the above definition is that $0 \in \sigma(S)$. The spectrum of $S$ is at most countable with 0 as the only possible accumulation point, [1].

The next two theorems can be found in [1].

Theorem 1.4. Let $X$ be a Banach space. The collection of all strictly singular operators is a closed subspace in $L(X)$, which is also a two-sided ideal.

Proposition 1.5. Let $X$ be a real Banach space and $X_{\mathbb{C}}$ its complexification. $A$ bounded operator $T: X \rightarrow X$ is strictly singular if and only if $T_{\mathbb{C}}: X_{\mathbb{C}} \rightarrow X_{\mathbb{C}}$ is strictly singular.

We show now that a certain class of operators on a complex Banach space can not be $J$-class. For this purpose we need the following proposition.

Proposition 1.6. Let $X$ be a complex Banach space and $T \in L(X)$. Suppose $\left(J_{T}(x)\right)^{\circ} \neq \emptyset$ for some $x \in X \backslash\{0\}$. Then $\sigma(T) \cap \partial \mathbb{D} \neq \emptyset$.

Proof. Assume that $\sigma(T) \cap \partial \mathbb{D}=\emptyset$. We decompose $\sigma$ in $\sigma_{1}:=\{\lambda \in \sigma:|\lambda|>1\}$ and $\sigma_{2}:=\{\lambda \in \sigma:|\lambda|<1\}$. Then $\sigma_{1}$ and $\sigma_{2}$ are disjoint and closed. By the Riesz decomposition theorem we can decompose $X=M_{1} \oplus M_{2}$, where $M_{1}$ and $M_{2}$ are closed and $T$-invariant subspaces and $\sigma_{1}=\sigma\left(T_{\mid M_{1}}\right), \sigma_{2}=\sigma\left(T_{\mid M_{2}}\right)$. Assume now that there exists a non-zero vector $x \in X$, such that $J_{T}(x)$ has non-empty interior. We can write $x=x_{1}+x_{2}$, with $x_{1} \in M_{1}$ and $x_{2} \in M_{2}$. Since $x \neq 0$, either $x_{1}$ or $x_{2}$ is not equal to zero. Consider the projection $P_{1}: X \rightarrow M_{1}$ along $M_{2}$ onto $M_{1}$. Since $J_{T}(x) \subset J_{\left.T\right|_{M_{1}}}\left(x_{1}\right)+J_{\left.T\right|_{M_{2}}}\left(x_{2}\right)$ it follows that $P_{1}\left(J_{T}(x)\right) \subset$ $J_{\left.T\right|_{M_{1}}}\left(x_{1}\right)$. By the open mapping theorem we get that $P_{1}\left(J_{T}(x)\right)$ has non-empty interior and hence $\left(J_{\left.T\right|_{M_{1}}}\left(x_{1}\right)\right)^{\circ} \neq \emptyset$. From the spectral radius formula we obtain that $\left\|T_{1}^{n} \widetilde{x}\right\| \leq a^{n}\|\widetilde{x}\|$ for some $a \in \mathbb{R}$ with $0 \leq a<1$ and for all $\widetilde{x} \in M_{1}$. This implies that $J_{\left.T\right|_{M_{1}}}\left(x_{1}\right)=\{0\}$ and therefore $M_{1}=\{0\}$. So we get $x_{1}=0$. Again 
from the spectral radius formula we know that $\left\|T_{1}^{n}(\hat{x})\right\| \geq A^{n}\|\hat{x}\|$ for some $A>1$ and all $\hat{x} \in M_{2}$. This implies $x_{2}=0$, which is a contradiction to our assumption that $x \neq 0$.

Theorem 1.7. Let $X$ be a complex infinite dimensional Banach space. Then for every operator of the form $T=\lambda I+S$, where $S$ is strictly singular and $|\lambda|>1$, and every $x \neq 0$, the set $J_{T}(x)$ has empty interior.

Proof. By Remark 1.3 it follows that $\lambda \in \sigma(T)$ and it is the only possible accumulation point. We decompose the spectrum in $\sigma_{1}:=\{\mu \in \sigma(T):|\mu| \leq 1\}$ and $\sigma_{2}:=\{\mu \in \sigma(T):|\mu|>1\}$. Clearly then $\lambda \in \sigma_{2}$. The set $\sigma_{1}$ is closed, and since $\lambda \in \sigma_{2}$, and $\lambda$ is the only possible accumulation point, we conclude that $\sigma_{2}$ is also closed. Futhermore $\sigma_{1}$ and $\sigma_{2}$ are disjoint. By the Riesz decomposition theorem we can decompose $X=M_{1} \oplus M_{2}$, where $M_{1}$ and $M_{2}$ are closed and $T$-invariant subspaces and $\sigma_{1}=\sigma\left(T_{\mid M_{1}}\right), \sigma_{2}=\sigma\left(T_{\mid M_{2}}\right)$. Now assume that there exists a nonzero vector $x$ with $\left(J_{T}(x)\right)^{\circ} \neq \emptyset$. Let $x=x_{1}+x_{2}$, where $x_{1} \in M_{1}$ and $x_{2} \in M_{2}$. Then since $x$ is non-zero, either $x_{1}$ or $x_{2}$ is not equal to zero. We claim that $M_{1}$ is finite dimensional. Otherwise suppose $M_{1}$ is infinite dimensional. Then $S_{\mid M_{1}}$ is strictly singular and it follows from Remark 1.3 that $0 \in \sigma\left(S_{\mid M_{1}}\right)$ and therefore $\lambda \in \sigma\left(T_{\mid M_{1}}\right)=\sigma_{1}$, which is not possible. Now consider the projection $P_{1}: X \rightarrow M_{1}$ along $M_{2}$ onto $M_{1}$. Since $J_{T}(x) \subset J_{T_{\mid M_{1}}}\left(x_{1}\right)+J_{T_{\mid M_{2}}}\left(x_{2}\right)$, it follows that

$$
P_{1}\left(J_{T}(x)\right) \subset J_{T_{\mid M_{1}}}\left(x_{1}\right) .
$$

By the open mapping theorem it follows that $P_{1}\left(J_{T}(x)\right)$ has non-empty interior and so $J_{T_{\mid M_{1}}}\left(x_{1}\right)$ has non-empty interior. This is possible only if $x_{1}=0$, since $M_{1}$ is finite dimensional; see [7.

As above we conclude that $J_{T_{\mid M_{2}}}\left(x_{2}\right)$ has non-empty interior. Therefore since $\sigma\left(T_{\mid M_{2}}\right)=\sigma_{2} \subset \mathbb{C} \backslash \overline{\mathbb{D}}$, it follows by Proposition 1.6 that $x_{2}=0$. Thus $x=x_{1}+x_{2}=$ 0 , which is a contradiction.

S. Argyros, A. Arvanitakis and A. Tolias constructed a non-separable real Banach space, on which every operator $T$ has the form $T=\lambda I+S$, where $S$ is strictly singular and has separable range; see $[3]$.

Theorem 1.8 (Argyros, Arvanitakis, Tolias). There exists a real non-separable Banach space $X_{A}$, containing no reflexive subspace, on which every operator $T$ is of the form $T=\lambda I+S$ with $\lambda \in \mathbb{R}$ and $S$ a weakly compact operator (and hence of separable range).

The fact that $X_{A}$ contains no reflexive subspace implies that every weakly compact operator on $X_{A}$ is strictly singular; thus every operator $T: X_{A} \rightarrow X_{A}$ is of the form $T=\lambda I+S$ with $\lambda \in \mathbb{R}$ and $S$ a strictly singular operator with separable range. The proof of the following corollary is essentially contained in the proof of Lemma 4.3 in 9 .

Corollary 1.9. Consider $X:=\left(X_{A}\right)_{\mathbb{C}}$. Then every operator $T \in L(X)$ is of the form $T=w I+S(w \in \mathbb{C})$, where $S$ is strictly singular and has separable range.

Proof. Every operator $T \in L(X)$ can be written as $T=T_{1}+i T_{2}$, where $T_{1}, T_{2} \in$ $L\left(X_{A}\right)$. By the previous theorem, $T_{1}=\lambda I+S_{1}$ and $T_{2}=\mu I+S_{2}(\lambda, \mu \in \mathbb{R})$, where 
$S_{1}, S_{2} \in L\left(X_{A}\right)$ are strictly singular and have separable range. Therefore we get

$$
\begin{aligned}
T & =T_{1}+i T_{2} \\
& =\lambda I+S_{1}+i\left(\mu I+S_{2}\right) \\
& =(\lambda+i \mu) I_{\mathbb{C}}+\left(S_{1}\right)_{\mathbb{C}}+i\left(S_{2}\right)_{\mathbb{C}} .
\end{aligned}
$$

By Proposition 1.5, $\left(S_{i}\right)_{\mathbb{C}}$ is strictly singular for $i \in\{1,2\}$ and by Theorem 1.4, $S:=\left(S_{1}\right)_{\mathbb{C}}+i\left(S_{2}\right)_{\mathbb{C}}$ is strictly singular and has separable range. With $w:=\lambda+i \mu$ we get $T=w I+S$.

The next lemma can be found in [7].

Lemma 1.10. Let $X$ be a Banach space and $T \in L(X)$. If $J_{T}(x)$ has non-empty interior for some $x \neq 0$, then $T-\lambda I$ has dense range for each $|\lambda| \leq 1$.

Theorem 1.11. There exists a non-separable complex Banach space $X$ on which the $J$-set of every operator has empty interior for every non-zero vector. In particular there does not exist a $J$-class operator on $X$.

Proof. We condsider the space $X=\left(X_{A}\right)_{\mathbb{C}}$. Then every operator $T$ is of the form $T=\lambda I+S$ by Corollary 1.9, where $S$ is strictly singular and has separable range. If $|\lambda|>1$, then it follows by Theorem 1.7 that the interior of $J_{T}(x)$ is empty for each non-zero vector $x$. Now consider $|\lambda| \leq 1$. Then by Lemma 1.10 the operator $T-\lambda I=S$ has dense range. This is not possible since $S$ has separable range and $X$ is non-separable.

Our next aim is to show that on the space $Y:=X \oplus X$, where $X=\left(X_{A}\right)_{\mathbb{C}}$, the $J$-set of every $T \in L(Y)$ also has empty interior for each non-zero vector in $Y$. The next lemma gives us some information about the form of the operators in $L(Y)$.

Lemma 1.12. Consider $Y:=X \oplus X$, where $X=\left(X_{A}\right)_{\mathbb{C}}$. Then for every operator $T \in L(Y)$ there exists an isomorphism $J \in L(Y)$, such that $J^{-1} T J$ has one of the following two matrix representations:

or

$$
J^{-1} T J=\left(\begin{array}{cc}
\lambda I & I \\
0 & \lambda I
\end{array}\right)+\left(\begin{array}{cc}
S_{1} & S_{2} \\
S_{3} & S_{4}
\end{array}\right)
$$

$$
J^{-1} T J=\left(\begin{array}{cc}
\lambda_{1} I & 0 \\
0 & \lambda_{2} I
\end{array}\right)+\left(\begin{array}{cc}
S_{1} & S_{2} \\
S_{3} & S_{4}
\end{array}\right)
$$

where $S_{i} \in L(X)$ are strictly singular and have separable range for $i \in\{1,2,3,4\}$.

Proof. Every operator $T \in L(Y)$ has the following matrix representation:

$$
T=\left(\begin{array}{ll}
T_{1} & T_{2} \\
T_{3} & T_{4}
\end{array}\right)
$$

where $T_{i} \in L(X)$ for $i \in\{1,2,3,4\}$. From Corollary 1.9 every $T_{i}=a_{i} I+\widetilde{S}_{i}$, where $\widetilde{S}_{i}$ is strictly singular with separable range. So we get

$$
T=\overbrace{\left(\begin{array}{ll}
a_{1} I & a_{2} I \\
a_{3} I & a_{4} I
\end{array}\right)}^{A}+\overbrace{\left(\begin{array}{ll}
\widetilde{S_{1}} & \widetilde{S_{2}} \\
\widetilde{S_{3}} & \widetilde{S_{4}}
\end{array}\right)}^{\widetilde{S}} .
$$


Applying the Jordan decomposition of matrices, there exists an isomorphism such that

$$
J^{-1} A J=\left(\begin{array}{cc}
\lambda I & I \\
0 & \lambda I
\end{array}\right) \text { or } J^{-1} A J=\left(\begin{array}{cc}
\lambda_{1} I & 0 \\
0 & \lambda_{2} I
\end{array}\right) .
$$

By Theorem 1.4, $S:=J^{-1} \widetilde{S} J$ is also strictly singular and therefore there exist some $S_{i} \in L(X), i \in\{1,2,3,4\}$ strictly singular with separable range (see [1]) such that

$$
S=\left(\begin{array}{ll}
S_{1} & S_{2} \\
S_{3} & S_{4}
\end{array}\right)
$$

The desired statement now follows.

The next theorem can be found in 1 .

Theorem 1.13. Assume that $S \in L(X)$ is strictly singular and that an operator $T \in L(X)$ has an at most countable spectrum. Then the spectrum of $S+T$ is at most countable and zero and the points of $\sigma(T)$ are the only possible accumulation points of $\sigma(S+T)$.

Theorem 1.14. Consider $Y=X \oplus X$ with $X=\left(X_{A}\right)_{\mathbb{C}}$ and $T \in L(Y)$. Then $\left(J_{T}((x, y))\right)^{\circ}=\emptyset$ for $(x, y) \in Y \backslash\{(0,0)\}$.

Proof. We argue by contradiction. So suppose there exists a $T \in L(Y)$ such that $\left(J_{T}((x, y))\right)^{\circ} \neq \emptyset$ for some $(x, y) \in Y \backslash\{(0,0)\}$. By Lemma 1.12 there exists an isomorphism $D \in L(Y)$ such that

$$
D^{-1} T D=\left(\begin{array}{cc}
\lambda I & I \\
0 & \lambda I
\end{array}\right)+\left(\begin{array}{cc}
S_{1} & S_{2} \\
S_{3} & S_{4}
\end{array}\right)
$$

or

$$
D^{-1} T D=\left(\begin{array}{cc}
\lambda_{1} I & 0 \\
0 & \lambda_{2} I
\end{array}\right)+\left(\begin{array}{cc}
S_{1} & S_{2} \\
S_{3} & S_{4}
\end{array}\right) \quad(* *)
$$

Then for $\widetilde{T}:=D^{-1} T D$ the $J$-set $J_{\widetilde{T}}\left(D^{-1}(x, y)\right)$ also has non-empty interior.

Case 1. $\widetilde{T}=(*)$.

If $|\lambda| \neq 1$, then we decompose $\sigma(\widetilde{T})$ in $\sigma_{1}=\{\mu \in \sigma(\widetilde{T}):|\mu|=1\}$ and $\sigma_{2}=$ $\{\mu \in \sigma(\widetilde{T}):|\mu| \neq 1\}$. The set $\sigma_{1}$ is closed and by Theorem 1.13, $\sigma_{2}$ is also closed, since $\lambda$ and 0 are the only possible accumulation points of $\sigma(\widetilde{T})$ and hence of $\sigma_{2}$. Furthermore the corresponding $\widetilde{T}$-invariant closed subspace $M_{1}$ for $\sigma_{1}$, resulting from the Riesz decomposition theorem is finite dimensional; otherwise,

$$
\left.\widetilde{T}\right|_{M_{1}}-\left.\left(\begin{array}{cc}
\lambda I & 0 \\
0 & \lambda I
\end{array}\right)\right|_{M_{1}}=\left.\left(\begin{array}{cc}
S_{1} & I+S_{2} \\
S_{3} & S_{4}
\end{array}\right)\right|_{M_{1}}
$$

is not invertible and hence $\lambda \in \sigma\left(\left.\widetilde{T}\right|_{M_{1}}\right)=\sigma_{1}$, which is not possible. The rest of the proof for $|\lambda| \neq 1$ is similar to Theorem 1.7.

Now consider $|\lambda|=1$. Then $\widetilde{T}-\left(\begin{array}{cc}\lambda I & 0 \\ 0 & \lambda I\end{array}\right)$ does not have dense range, which is a contradiction to Lemma 1.10 . 
Case 2. $\widetilde{T}=(* *)$.

If $\lambda_{1}=\lambda_{2}$ the argumentation is almost similar to case 1 . So suppose $\lambda_{1} \neq$ $\lambda_{2}$. Assume $\left|\lambda_{1}\right|=1$ or $\left|\lambda_{2}\right|=1$. Without loss of generality $\left|\lambda_{1}\right|=1$. Then $\widetilde{T}-\left(\begin{array}{cc}\lambda_{1} I & 0 \\ 0 & \lambda_{1} I\end{array}\right)$ does not have dense range, which is a contradiction as in Case 1.

For $\left|\lambda_{1}\right| \neq 1$ and $\left|\lambda_{2}\right| \neq 1$ the argumentation is identical to Case 1.

Remark 1.15. It is also possible with some more technicalities to prove the same result in Theorem 1.14 for $Y=\overbrace{X \oplus \ldots \oplus X}^{n \text { times }}$, where $X=\left(X_{A}\right)_{\mathbb{C}}$.

We will now show that there is a large class of non-separable Banach spaces on which there always exists a $J$-class operator, namely the reflexive non-separable Banach spaces. The next theorem can be found in [7].

Theorem 1.16. Let $X$ be a Banach space and $Y$ a separable Banach space. Consider $S \in L(X)$ with $\sigma(S) \subset\{\lambda:|\lambda|>1\}$. Also let $T \in L(Y)$ be hypercyclic. Then:

(1) $S \times T: X \times Y \rightarrow X \times Y$ is a J-class operator, but not hypercyclic.

(2) $A_{S \times T}=\{0\} \times Y$.

The next theorem by Lindenstrauss ([8]) gives us some information about the decomposition of reflexive non-separable Banach spaces.

Theorem 1.17 (Lindenstrauss). Let $X$ be a non-separable reflexive Banach space and $Y \subset X$ a separable and closed subspace. Then there exists a separable closed subspace $W$ of $X$ that contains $Y$ and a linear bounded projection $P_{W}: X \rightarrow W$ with $\left\|P_{W}\right\|=1$.

Theorem 1.18. Let $X$ be a non-separable reflexive Banach space. Then for every infinite dimensional separable and closed subspace $Y$ and for every $\lambda \in(1, \infty)$ there exists a $J$-class operator $T$ with $Y \subset A_{T}$ and $\|T\|=\lambda$.

Proof. By Theorem 1.17 there exists a separable infinite dimensional subspace $W$ that contains $Y$ and a linear bounded projection $P_{W}: X \rightarrow W$ with $\left\|P_{W}\right\|=1$. There exists a closed subspace $U$ of $X$ such that $X=U \oplus W$. For given $\epsilon>0$ we can find a hypercyclic operator $T_{1}: W \rightarrow W, T_{1}:=I_{W}+K$, with $K$ compact and $\|K\|<\epsilon$; see $\left([2]\right.$, 6]). Then by Theorem 1.16 the operator $T_{\lambda}:=\lambda I_{U} \oplus T_{1}=$ $\lambda I+(1-\lambda) P_{W}+K \circ P_{W}$ is $J$-class for $\lambda>1$. Furthermore $Y \subset W=A_{T}$. Now define the function $g:(1, \infty) \rightarrow \mathbb{R}$ by $g(\delta):=\left\|T_{\delta}\right\|$. Then it is easy to see that $g$ is continuous. For given $\lambda$ we choose $\delta>1$ and $\epsilon>0$ such that $2 \delta+\epsilon<1+\lambda$. Therefore we get

$$
\begin{aligned}
g(\delta) & =\left\|T_{\delta}\right\|=\left\|\delta I+(1-\delta) P_{W}+K \circ P_{W}\right\| \\
& \leq \delta+|1-\delta|\left\|P_{W}\right\|+\left\|P_{W}\right\|\|K\| \leq 2 \delta-1+\epsilon<\lambda .
\end{aligned}
$$

On the other hand we can find a $\mu>1$ large enough such that $g(\mu)>\lambda$. By the intermediate value theorem there exists a $\xi \in[\delta, \mu]$ with $g(\xi)=\left\|T_{\xi}\right\|=\lambda$.

\section{ACKNOWLEDGEMENTS}

The author is grateful to Prof. Dr. Rainer Brück for his scientific support. He would also like to express his gratitude to Professor Dr. W. Kaballo for some helpful comments. 


\section{REFERENCES}

[1] Y. A. Abramovich, C. D. Aliprantis, An Invitation to Operator Theory, Amer. Math. Soc. (Graduate Studies in Mathematics, V. 50), 2002. MR1921782 (2003h:47072)

[2] S. I. Ansari, Existence of Hypercyclic Operators on Topological Vector Spaces, J. Funct. Anal. 148 (1997), no. 2, 384-390. MR.1469346 (98h:47028a)

[3] S. Argyros, A. Arvanitakis, A. Tolias, Saturated Extensions, The Attractors Method and Hereditarily James Tree Spaces, Methods in Banach space theory, 1-90, London Math. Soc. Lecture Note Ser., 337, Cambridge Univ. Press, Cambridge, 2006. MR2326379 (2009d:46021)

[4] M. R. Azimi, V. Müller, A note on J-sets of linear operators, Revista de la Real Academia de Ciencias Exactas, Fisicas y Naturales 105 (2) (2011), 449-453. MR2826722

[5] T. Bermudez, N. J. Kalton, The range of operators on von Neumann algebras, Proc. Amer. Math. Soc. 130 (2002), no. 5, 1447-1455. MR.1879968(2003a:47078)

[6] L. Bernal-Gonzales, On hypercyclic operators on Banach spaces, Proc. Amer. Math. Soc. 127 (1999), no. 4, 1003-1010. MR.1476119 (99f:47010)

[7] G. Costakis, A. Manoussos, J-class operators and hypercyclicity, J. Operator Theory, to appear.

[8] J. Lindenstrauss, On non-separable reflexive Banach spaces, Bull. Amer. Math. Soc. 72 (1966), no. 6, 967-970. MR0205040 (34:4875)

[9] S. Shkarin, On the spectrum of frequently hypercyclic operators, Proc. Amer. Math. Soc. 137 (2009), no. 1, 123-134. MR2439433 (2009g:47020)

Fakultät für Mathematik, Technische Universität Dortmund, D-44221 Dortmund, Germany

E-mail address: amirbahman@hotmail.de 